\title{
LEACH-C Variants using Different Optimization Techniques: A Survey
}

\author{
Nidhi Saini \\ M.Tech scholar \\ DAVIET \\ Kabir Nagar, Jalandhar
}

\author{
Rajeev Kumar \\ Assistant Professor (IT) \\ DAVIET \\ Kabir Nagar, Jalandhar
}

\author{
Reeta Bhardwaj \\ Assistant Professor (IT) \\ DAVIET \\ Kabir Nagar, Jalandhar
}

\begin{abstract}
Low Energy Efficient Clustering Hierarchy-Centralised (LEACHC) protocol is one of the most pivotal parts of Wireless Sensor Networks (WSN). LEACH-C protocol employs Simulated Annealing algorithm, which is an optimization technique for cluster-head $(\mathrm{CH})$ selection as well as cluster formation. A number of LEACH-C variants came into consideration that uses different optimization techniques in place of Simulated Annealing for $\mathrm{CH}$ selection, cluster formation and data transmission also. This paper provides an insight to such LEACH-C based protocols and also compare their performance in terms of network lifetime, stability period and energy consumption in a tabular form. Network lifetime is compared by evaluating the number of nodes dead with respect to the rounds.
\end{abstract}

\section{Keywords}

Cluster; Cluster-head; data transmission; energy consumption; LEACH-C; network lifetime; optimization; simulated annealing; stability period; Wireless Sensor Networks

\section{INTRODUCTION}

WSN is one of the most explored research subjects in the field of computer science. WSN makes use of sensor nodes which are small sized devices deployed at remote locations without any physical connection for collecting the information from their surroundings and transmit it for the purpose of evaluation and generating required data. These sensor nodes are capable of detecting variety of physical parameters such as light, sound, temperature, air, pressure, any activity taking place in the surroundings, attributes of an object such as height, size, speed and many more. Sensor nodes are mostly battery-operated, which gets consumed up at a very fast rate. Faster power consumption of the sensor nodes is the most alarming issue in WSN. A huge count of improvisions is made and protocols are built for WSN. These sensor nodes consume less power, have low cost, small in size, battery powered and are multifunctional nodes that can be deployed anywhere easily.[1] WSN is often used in the civilian as well as military field for the purpose of monitoring far away locations like border areas, battlefield, remote sensing, intelligent guiding, protection of confidential places like military headquarters, nuclear plants and many more.

Low Energy Adaptive Clustering Hierarchy (LEACH) [2] protocol has proved to be a milestone in the field of WSN, which was further improved by its authors and they proposed another protocol LEACH-C [3]. In LEACH-C protocol, Simulated annealing [4] algorithm is used for the purpose of cluster formation and $\mathrm{CH}$ selection and provides much better results as compared to the former protocol. Optimization is the technique used for selecting the best solution among the given set of candidate solutions. It gives brilliant results for NP-hard problems.
There are large number of problems related to optimization in computer science which needs to be solved as efficiently as possible. Some of these problems are Travelling Salesman Problem (TSP) [5], knapsack problem [6], vehicle routing and nQueen problem. In global optimization, solution must have minimum value for the objective function of the problem. Enormous strategy and tactics have been proposed till now to solve these NP-hard problems with efficiency to get the best possible results. Most of the optimization techniques are inspired from the nature and also tend to provide best results.

The remaining paper is organised as follows: Section 2 illustrates the related work, Section 3 consists of detailed information about the LEACH-C variants using optimization techniques, Section 4 compares the protocols, Section 5 gives the results of the comparisons and Section 6 concludes the paper.

\section{RELATED WORK}

LEACH (Low Energy Adaptive Clustering Hierarchy) is a protocol architecture developed by Wendi Heinzelman, Anantha Chandrakasan and Hari Balakrishnan in 2000 [2]. In a network of nodes capable of sensing the environment and collecting the data, the data collected is often inter-related. There is a BS in the network to which this data should be transmitted. If each node will send data individually to the BS, this will result in the heavy traffic in the network. Also the data collected by these nodes is corelated, the BS may get same data from different nodes which are located near to each other. Such situations can be avoided by using LEACH protocol architecture. LEACH protocol solves this problem by forming the clusters of existing nodes and each cluster consists of a $\mathrm{CH}$. All the nodes are distributed into different clusters. The $\mathrm{CH}$ acts like a BS for the particular cluster. All the nodes present in a cluster will send their data to this $\mathrm{CH}$. Further operations on the data will be made by this $\mathrm{CH}$ only. Fig. 1 illustrates the formation of clusters using sensor nodes in LEACH in WSN.

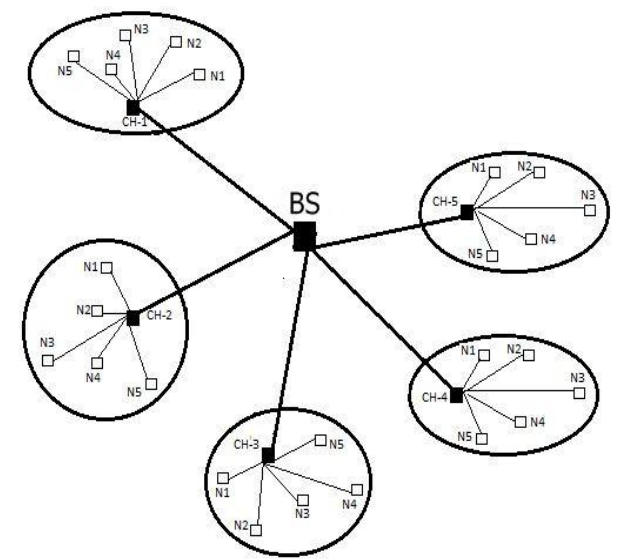

Fig. 1: Clustering in LEACH 
Optimization means selecting the best solution from the given set of candidate solutions. A number of meta-heuristics are proposed for this purpose. Optimization techniques are categorized into two kinds as mentioned in fig 2. First, single solution based algorithms are based on the technique of selecting a random candidate solution and then improving it step by step, like Simulated annealing and Simulated Raindrop algorithm [7]. On the other hand, population based meta-heuristics depends upon exploring the best solution from the given set of solutions.

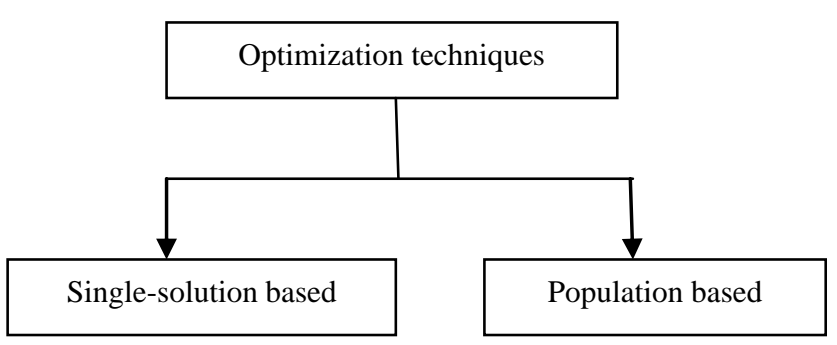

Fig. 2: Categories of optimization techniques

\section{LEACH-C AND ITS VARIANTS USING OPTIMIZATION TECHNIQUE}

A number of LEACH-C variants are proposed that make use of optimization techniques.

\subsection{Low Energy Adaptive Clustering Hierarchy- Centralized (LEACH-C)}

LEACH-C is a protocol architecture developed by Wendi Heinzelman, Anantha Chandrakasan and Hari Balakrishnan in 2002 [3]. It is an improved version of LEACH protocol architecture. It is application specific protocol architecture for Wireless Microsensor networks. [1] It is quite similar to LEACH protocol. It also consists of same phases. In fact, there is no change in the steady-state phase. The only change took place is in set-up phase to form clusters.

The major difference between LEACH and LEACH-C protocol architecture is the method used for forming clusters. In LEACH, distributed algorithm is used for this purpose while LEACH-C makes use of simulated annealing algorithm. This approach has given better results. This central clustering technique gives better results due to formation of better clusters and $\mathrm{CH}$ nodes are dispersed throughout the network. Moreover, in LEACH there is no guarantee of the placement or number of $\mathrm{CHs}$ in the network. $\mathrm{CHs}$ may be close to each other, which limits the quality of the results.

LEACH-C also performs its functions in the set of rounds, like LEACH. Each round consists of two phases, namely, set- up phase and steady-state phase.

Set up phase: In the set up phase of LEACH-C, each node needs to transmit information about their location to the BS of the network. The information about the location of node is determined by using Global positioning system (GPS) receiver in each round. Next step is to form the clusters of these nodes, which is referred to as a NP-hard problem. The most reliable method of solving NPhard problems is to use approximation algorithms to determine optimal solution of the problem in polynomial time [8]. In LEACH-C protocol architecture, simulated annealing algorithm [4] is used, which is based on the principle of thermodynamics. This algorithm will form clusters for LEACH protocol by determining whether a node will belong to the current cluster being formed or not. This algorithm makes use of probabilistic functions to determine the optimal result for NP-hard problem. The first step is to ensure that all the nodes in the network have approximate equal energy. To ensure this, average node energy is calculated and then compared with the individual nodes. In case, any node have lesser energy than the average it cannot be the $\mathrm{CH}$ in the present round. Number of optimal clusters is found by using equation $1[3]$.

$$
\mathrm{k}_{\text {optimized }}=\sqrt{\frac{\mathrm{N}}{2 \pi} * \frac{\epsilon \mathrm{fs}}{\varepsilon \mathrm{mp}}} * \frac{\mathrm{M}}{\mathrm{d}_{\mathrm{toBS}}^{2}}
$$

where kopt is optimal number of clusters, $\mathrm{N}$ is number of sensor nodes in the network, $\mathrm{M}$ is region of network, dtoBS is average distance to base station from sensor nodes, Efs is energy consumed by using free-space model and Emp is energy consumed for transmission by using multipath model.

Now simulated annealing algorithm will determine the probability of the nodes to become $\mathrm{CH}$ in the network using equation 2 [8] and then associates them to their respective clusters. $\mathrm{CHs}$ are selected such that they are dispersed in the network and the nodes in the cluster are too close to them. Thus, the squared distance between $\mathrm{CHs}$ and nodes is kept minimum which also results in the lesser energy consumption of the non $\mathrm{CH}$ nodes to transmit collected data to the $\mathrm{CHs}$.

$$
\begin{gathered}
\mathrm{p}_{\mathrm{k}}=\left\{\begin{array}{cc}
\mathrm{e}^{\frac{-\left(\mathrm{f}\left(\mathrm{C}^{1}\right)-\mathrm{f}(\mathrm{C})\right)}{\mathrm{a}_{\mathrm{k}}}} & \mathrm{f}\left(\mathrm{C}^{1}\right) \geq \mathrm{f}(\mathrm{C}) \\
1 & \mathrm{f}\left(\mathrm{C}^{1}\right)<f(C)
\end{array}\right. \\
\mathrm{f}(\mathrm{C})=\sum_{\mathrm{i}=1}^{\mathrm{N}} \min ^{2}(\mathrm{i}, \mathrm{c})
\end{gathered}
$$

After the formation of both $\mathrm{CH}$ nodes and clusters, this information is broadcast to all the nodes by the BS. The BS broadcasts a message consisting of the CH ID. Each node checks this ID and compares with their own ID. If the ID matches, that node takes up the responsibility of the $\mathrm{CH}$. This TDMA schedule consists of different slot for each node in the cluster when it can send its data. All non $\mathrm{CH}$ nodes will determine their TDMA schedule to transmit data to $\mathrm{CH}$ and goes to the sleep state. This prevents unnecessary loss of energy of non $\mathrm{CH}$ nodes.

Steady-state phase: The steady-state phase of LEACH-C is exactly identical to that of LEACH protocol. All the nodes in the network sense their environment and record various events taking place. The nodes will send this collected data to their respective $\mathrm{CHs}$ in the TDMA slot assigned to them. The receiver of non $\mathrm{CH}$ nodes activate only during this allocated time slot to conserve the energy of the nodes. If a node is unable to send its complete data in one slot, it can resume it the next time on arriving its slot. The receiver of $\mathrm{CH}$ always remains on in this phase to get data from different nodes. The operations of the $\mathrm{CHs}$ require more energy and also their energy is consumed too fast. But this energy consumption is less as compared to that in LEACH because of the lesser distance between $\mathrm{CH}$ and non $\mathrm{CH}$ nodes. After receiving the data, the $\mathrm{CH}$ evaluates it and determine the required information which is to be sent to the BS by compressing into a single signal. This prevents unnecessary traffic in the network as only few nodes have to send data to the BS.

\subsection{Balanced Clustering Energy Efficient Hierarchical Routing (BCEE)}

BCEE [9] is a protocol proposed by Cui and Liu. It makes use of k-means clustering algorithm for forming clusters in the WSN and Ant Colony Optimization (ACO) [10] for routing data from sensor nodes to the $\mathrm{CH}$. This algorithm works in two phases. The first phase includes the formation of balanced clusters in the network though sensor nodes are not positioned exactly but they made use of RSSI (Received Signal Strength Indicator). The second phase includes routing the data from $\mathrm{CH}$ nodes to base station by 
optimizing the power consumption during the process. ACO is one of the best population-based optimization techniques that provide the best results for routing. This protocol has given significant improvement in energy efficiency in comparison to LEACH-C protocol. The lifespan of the sensor nodes has also enhanced to the great extent. This protocol was enhanced further using modified ACO algorithm in LEACH-C.

\subsection{Particle Swarm Optimization-Centralized (PSO-C)}

Latiff et al. has proposed an Energy aware clustering protocol [11] that makes use of Particle Swarm Optimization (PSO) [12] technique for creation of clusters in WSN. The BS employs PSO to determine $\mathrm{CH}$ nodes which minimizes the cost function mention in equation 4.

$$
f=\alpha * f_{1}+(1-\alpha) * f_{2}
$$

Where $\alpha$ is the control parameter, $\mathrm{f}_{1}$ is the function that determines maximum average Euclidean distance between node and the $\mathrm{CH}$ node and $\mathrm{f}_{2}$ is the total initial energy in network to the total energy of $\mathrm{CH}$ nodes in the current round.

Apart from this cost function, rest of the working of the protocol is similar to [3]. This protocol have much better performance as compared to former LEACH-C protocol especially in the terms of network lifetime and successful data packet transmission to the BS. The clusters are well-formed due to evenly positioning of $\mathrm{CH}$ nodes in the network which also reduces the energy consumption of the energy consumption of the nodes.

\subsection{Low Energy Adaptive Clustering Hierarchy- Genetic Algorithm (LEACH-GA)}

Liu et al. has proposed a new protocol named LEACH-GA [13] in 20011 that uses of optimization based Genetic Algorithm [14] for $\mathrm{CH}$ selection in the network. It consists of three phases. First phase is the preparation phase which determines the possible $\mathrm{CH}$ nodes in the network. In subsequent rounds there are only two phases, namely, set-up phase and steady-state phase. Firstly, every node in the network determines whether it is a possible $\mathrm{CH}$ node by performing a $\mathrm{CH}$ selection process defined in this protocol. The probability to become a $\mathrm{CH}$ node is evaluated using the equation 5 .

$$
p(i)=\frac{k_{\text {optimized }}}{n}
$$

Where $\mathrm{k}_{\text {optimized }}$ is the number of clusters to be formed in the network and $\mathrm{n}$ signifies the number of rounds.

The node transmits their respective message to the Base Station (BS) in the network along with their node ID and position details. The BS performs an optimization to calculate probability of that node to be $\mathrm{CH}$ by Genetic Algorithm method, which aims to decline energy consumption of sensor nodes. The BS again broadcast an advertisement message to all the nodes [15] along with their probability so that they can form the clusters. The other two phases are performed in the same way as that in LEACH protocol. There are some other optimization techniques that give much better results as compared to Genetic algorithm.

\subsection{Particle Swarm Optimization-Semi distributed (PSO-SD)}

Singh et al. make use of PSO [12] technique to make selection of $\mathrm{CH}$ nodes in the network. A novel fitness function is determined for execution of cluster based PSO which is based on the distance, degree of node, energy and head count of the sensor nodes in a cluster [15]. This fitness function defines the optimized location of $\mathrm{CH}$. The results provided by this approach are much better than LEACH and some other comparatives on the basis of lifetime of nodes and energy consumption. They have proposed a fitness function as mentioned in equation 6 [15].

$$
\begin{aligned}
& f\left(x_{i}(t)\right)=\text { optimize }\left(\beta_{1} X_{1}+\beta_{2} X_{2}+\beta_{3} X_{3}+\left(1-\beta_{1}-\right.\right. \\
& \left.\left.\beta_{2}-\beta_{3}\right) X_{4}\right)
\end{aligned}
$$

Where $\mathrm{f}\left(\mathrm{x}_{\mathrm{i}}(\mathrm{t})\right)$ is the fitness function for $\mathrm{i}^{\text {th }}$ node, $\beta_{1}, \beta_{2}, \beta_{3}$ are the control parameters used to provide weightage to different factors used to determine fitness function. $\mathrm{X}_{1}$ denotes the mean distance of the node from other member nodes of the cluster. $\mathrm{X}_{2}$ signifies the remaining energy of the node in comparison to other member nodes in the cluster. $X_{3}$ denotes the node degree [15].

PSO-SD is semi-distributed protocol and hence provides better results in comparison to former protocols.

\subsection{Low Energy Adaptive Clustering Hierarchy- Modified Ant Colony optimization (LEACH-MA)}

In this paper, Varha Gupta and Shashi Kumar Sharma [16] has used Modified Ant Colony Optimization algorithm in collaboration with LEACH protocol. Here, ACO was used to make selection of $\mathrm{CHs}$ in the network of sensor nodes. The pheromone value is determined for each node in the network based on its energy and the probability and determines probability based on the pheromone and distance from the $\mathrm{CH}$ node. Update the final $\mathrm{CHs}$ selected along with their pheromone values. This $\mathrm{CH}$ generates TDMA schedule for its member nodes for transmitting their collected data. Then the $\mathrm{CH}$ with maximum probability in the network is selected as $\mathrm{CH}$ Leader. Each $\mathrm{CH}$ sends data to this $\mathrm{CH}$ Leader which again transmits it to the BS. This approach gives an improved result in terms of energy consumption of nodes and lifetime of the network as compared to LEACH protocol. The probability of a node to become $\mathrm{CH}$ is computed using equation 7 .

$$
\begin{gathered}
p(i)=\frac{d(i) * \alpha+p h_{i}(t) * \beta}{\sum_{I=1}^{n} d(i) * \alpha+p h_{i}(t) * \beta} \\
p h_{i}(t)=\frac{T_{i j}^{\alpha} * n_{i}(t)^{\beta}}{\sum_{I=1}^{n} T_{i j}^{\alpha} * n_{i}(t)^{\beta}} \\
n_{i}(t)=\frac{1}{I e-e}
\end{gathered}
$$

Where $d_{i}$ is the distance of node from BS, $\alpha$ and $\beta$ are control parameters, $i$ is the $\mathrm{CH}$ node, $\mathrm{j}$ is the next node to be chosen as $\mathrm{CH}$, $T_{i j}$ is the pheromone intensity and $n_{i}$ is the heuristic function as defined in equation 9 [16] in which Ie is the initial energy level of the node and e is the actual energy level of the same node.

\section{COMPARISON}

Table 1 compares the LEACH-C and its variants that use optimization techniques. They are compared on the basis of Modification rule, motivation (technique used), energy efficiency, data transmission type and clustering method used for grouping of the clusters. 
Table 1: Comparison of LEACH-C variants using optimization techniques

\begin{tabular}{|c|c|c|c|c|c|c|}
\hline Year & Protocol & Modification & Motivation & $\begin{array}{c}\text { Energy } \\
\text { efficiency }\end{array}$ & $\begin{array}{c}\text { Data } \\
\text { transmission }\end{array}$ & $\begin{array}{c}\text { Clustering } \\
\text { method }\end{array}$ \\
\hline 2002 & LEACH-C & $\begin{array}{c}\text { CH selection } \\
\text { (energy efficiency) }\end{array}$ & $\begin{array}{c}\text { Simulated Annealing } \\
\text { Algorithm }\end{array}$ & High & Single hop & Centralised \\
\hline 2007 & PSO-C & Cluster formation & $\begin{array}{c}\text { Particle Swarm } \\
\text { Optimization }\end{array}$ & Very High & Single-hop & Centralised \\
\hline 2009 & BCEE & Data transmission & $\begin{array}{c}\text { Ant Colony Optimization } \\
\text { and K-means clustering } \\
\text { strategy }\end{array}$ & Very High & Multi-hop & Centralised \\
\hline 2011 & LEACH-GA & CH selection & Genetic Algorithms & High & Single-hop & Distributed \\
\hline 2013 & PSO-SD & $\begin{array}{c}\text { CH selection using } \\
\text { and forming clusters }\end{array}$ & $\begin{array}{c}\text { Particle Swarm } \\
\text { Optimization technique } \\
\text { is used to select CHs } \\
\text { and form energy-aware } \\
\text { clusters. }\end{array}$ & Very high & Single-hop & Semi- \\
\hline 2015 & LEACH-MA & CH selection & $\begin{array}{c}\text { Modified ACO } \\
\text { technique is used to } \\
\text { select the CHs. }\end{array}$ & High & Single-hop & Centralised \\
\hline
\end{tabular}

\section{RESULTS}

The protocols are compared on the basis of network lifetime by using the following parameters. Furthermore, these protocols are compared on the basis of their network lifetime. Table 2 provides statistics for LEACH-C, PSO-C, BCEE, LEACH-GA,
PSO-SD and LEACH-MA for the first node dead, 10\% nodes dead, 50\% nodes dead and all nodes dead with respect to number of rounds for which protocols are run. nodes in the network for different LEACH based protocols at different rounds.

Table 2: Number of nodes alive w.r.t. the rounds

\begin{tabular}{|l|c|c|c|c|c|c|}
\hline & LEACH-C & LEACH-MA & BCEE & PSO-SD & PSO-C & LEACH-GA \\
\hline First Node Dead & 1120 & 1201 & 1194 & 1213 & 1007 & 1167 \\
\hline 10\% Nodes Dead & 1247 & 1332 & 1301 & 1376 & 1312 & 1254 \\
\hline Half Nodes Dead & 1348 & 1443 & 1402 & 1456 & 1396 & 1374 \\
\hline 75\% Nodes Dead & 1435 & 1585 & 1536 & 1585 & 1498 & 1430 \\
\hline Last Node Dead & 1459 & 1865 & 1804 & 1934 & 1786 & 1732 \\
\hline
\end{tabular}

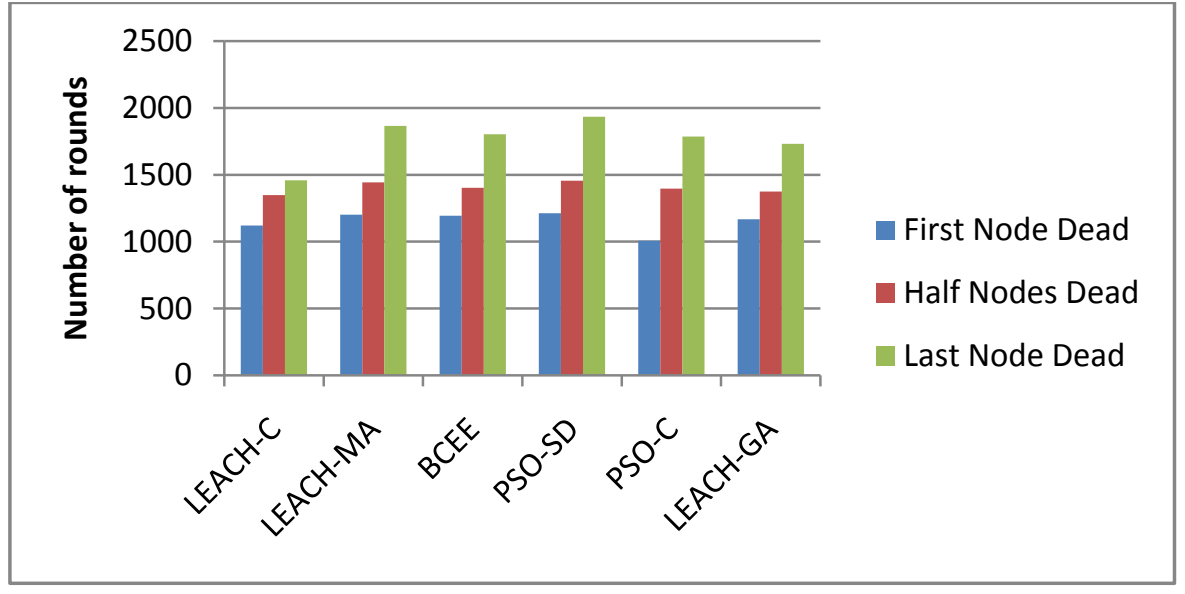

Fig 2: Number of rounds vs. Number of alive nodes 


\section{CONCLUSION}

In this paper, different LEACH-C based protocols have been analyzed that made use of different optimization techniques. The use of these techniques in the field of WSN and especially for LEACH has improved the performance of the networks to a great extent. Most of them are used for the purpose of $\mathrm{CH}$ selection and cluster formation. They provide better positioning of the $\mathrm{CH}$ nodes in the network which helps a lot in reducing the energy consumption of the sensor nodes. From these protocols PSO-SD provides the best results because it is semi-distributed protocol.

Latest optimization techniques like Simulated Raindrop Algorithm and Intelligent Water-drop Algorithm can be used to obtain better results. These techniques can be utilized for solving the NP-hard problems. Apart from this, Simulated Raindrop Algorithm can be replaced by Simulated Annealing in LEACH-C protocol which may result into a much efficient $\mathrm{LEACH}-\mathrm{C}$ variant.

\section{REFERENCES}

[1] Jun Zheng and Abbas Jamalipour. Wireless Sensor Networks - A Networking Perspective. IEEE, Book, A John Wiley \& Sons INC. Publication.

[2] Wendi Rabiner Heinzelman, Anantha Chandrakasan and Hari Balakrishnan. 2000. Energy Efficient Communication Protocol for Wireless Microsensor Networks. IEEE- Proceedings of the 33rd Hawaii International Conference on System Sciences.

[3] Wendi Rabiner Heinzelman, Anantha Chandrakasan and Hari Balakrishnan. 2002. An Application-Specific Protocol Architecture for Wireless Microsensor Networks IEEE- IEEE Transactions on Wireless Communications, Vol. 1, No. 4, pp. 660-670.

[4] Scott Kirkpatrick. 1984. Optimization by Simulated Annealing: Quantitative Studies. Journal of Statistical Physics, Vol. 4, No. 5/6.

[5] S. Lin. 1965. Computer Solutions of the Travelling Salesman Problem. Bell Syst. Journal, vol. 44, pp. 2245 2269

[6] H. Shah-Hosseini. 2008. Intelligent water drops algorithm: a new optimization method for solving the multiple knapsack proble. Int. Journal of Intelligent Computing and Cybernetics, Vol. 1, No. 2, pp. 193-212, 2008a.
[7] Amin Ibrahim, Shahryar Rahnamayan and Miguel Vargas Martin. 2014. Simulated Raindrop Algorithm for Global Optimization. IEEE - 27th Canadian Conference on Electrical and Computer Engineering, pp. 1-8.

[8] Wendi Beth Heinzelman. 2000. Application-Specific Protocol Architecture for Wireless Networks. Thesis, MIT.

[9] Cui Xiaoyan, Liu Zhao. 2009. BCEE: a balancedclustering, energy-efficient hierarchical routing protocol in wireless sensor networks. Proceedings of ICNIDC2009. IEEE, 2009. p. 26-30

[10] Marco Dorigo, Christian Blum. 2005. Ant Colony Optimization Theory: A Survey. Theoretical Coimputer Science 344 pp. 243-278.

[11] N.M. Abdul Latiff, C.C. Tsimenidis, B.S. Sharif. 2007. Energy-Aware Clustering for Wireless Sensor Networks using ParticleSwarm Optimization. The 18th Annual IEEE International Symposium on Personal, Indoor and Mobile Radio Communications (PIMRC'07)

[12] Russell Eberhart, James Kennedy. 1995. A new optimizer using Particle Swarm Theory. IEEE Sixth International Symposium on Micro Machine and Human Science.

[13] Jenn-Long Liu and Chinya V. Ravishankar. 2011. Genetic Algorithm-Based Energy-Efficient Adaptive Clustering Protocol for Wireless Sensor Networks", International Journal of Machine Learning and Computing, Vol. 1, No. 1, pp. 79-85.

[14] J.H. Holand. 1992. Adaptation in natural and artificial systems: an introductory analysis with applications to biology, control, and artificial intelligence. MIT Press.

[15] Buddha Singh and Daya Krishan Lobiyal. 2012. A Novel-Energy Aware cluster head selection based on particle swarm optimization for wireless sensor networks. Springer- Human Centric Computing and Information Sciences.

[16] Varsha Gupta and Shashi Kumar Sharma. 2015. Cluster Head Selection using Modified ACO. Proceedings of $4^{\text {th }}$ International Conference on Soft Computing for Problem Solving, Advances in Intelligent systems and Computing,

2015. 